\title{
Analyse des textures cristallographiques et des microstructures
}

Thierry Baudin (1) (thierry.baudin@u-psud.fr), Daniel Chateigner ${ }^{(2)}$,

Claude Esling ${ }^{(3)}$, Luca Lutterotti ${ }^{(2,4)}$ et Magali Morales ${ }^{(5)}$

(1) Université Paris-Sud, SP2M, ICMM0, UMR CNRS 8182, 91405 Orsay Cedex

(2) CRISMAT-ENSICAEN et IUT-Caen, Université de Caen Basse-Normandie, Campus 2, 6 bd. Maréchal Juin, 14050 Caen

(3) Laboratoire LEM3, CNRS UMR 7239, et Laboratory of excellence DAMAS, Université de Lorraine, 57045 Metz

(4) Department of Materials Engineering, Engineering Faculty, University of Trento, 77 via Mesiano, 38123 Trento, Italie

(5) CIMAP-ENSICAEN, Université de Caen Basse-Normandie, 6 bd. Maréchal Juin, 14050 Caen

La texture cristalline,

c'est-à-dire l'orientation

cristallographique des grains, tout comme leur taille et leur

forme, sont des paramètres

qui déterminent les propriétés des matériaux polycristallins.

La diffraction des rayons $X$

ou des neutrons permet

d'accéder à cette texture,

alors que celle des électrons

rétrodiffusés dans

un microscope électronique

à balayage est à même

de caractériser simultanément

la microtexture

et la microstructure.

Ces différentes techniques

de caractérisation sont

succinctement décrites,

et plusieurs exemples sont

présentés afin d'illustrer

l'intérêt de la caractérisation

de la texture pour une bonne

maîtrise des propriétés

d'usage des matériaux.
Les cristallites des matériaux polycristallins peuvent posséder une ou des orientations préférentielles, compte tenu de la gamme de transformations thermomécaniques adoptée lors de leur élaboration et de leur mise en forme. Les propriétés d'usage de ces matériaux texturés dépendent de la taille et de la forme des cristallites et de leur orientation cristallographique. En effet, la texture joue un rôle crucial, par exemple, sur les propriétés d'emboutissabilité des tôles utilisées par l'industrie automobile. D'autres exemples seront décrits dans le cadre de cet article. On voit alors bien l'intérêt de quantifier les différentes composantes (orientations) de texture présentes dans le matériau, leur maîtrise permettant l'optimisation des propriétés d'emploi recherchées [1].

La quantification de la texture peut être faite à partir de mesures globales par diffraction des rayons $\mathrm{X}$ (analyse en surface) ou des neutrons (analyse en volume). On peut alors accéder, via le calcul de la fonction de distribution des orientations cristallines (FDOC), respectivement, aux fractions surfaciques ou volumiques des différentes composantes présentes dans la texture [2]. Cependant, ces approches ne permettent pas de préciser la répartition spatiale de ces orientations dans le polycristal. Pour ce faire, il est nécessaire d'utiliser des approches locales qui décrivent simultanément la microstructure (taille et forme des grains) et la microtexture (orientation cristallographique des grains). La diffraction des électrons rétrodiffusés (EBSD, Electron BackScatter Diffraction) dans

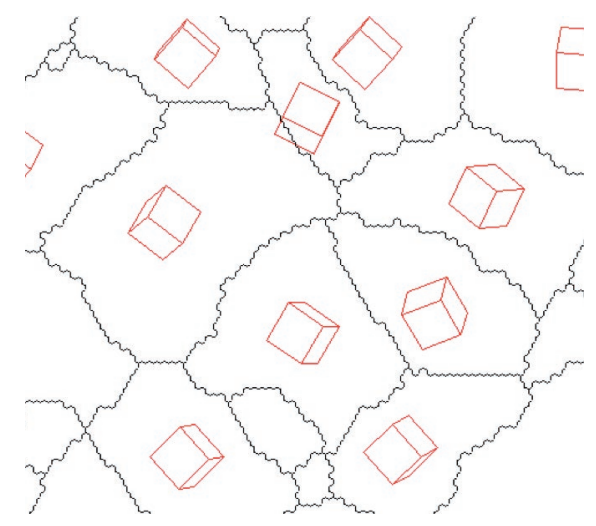

1. Description schématique de l'orientation des grains à partir de cubes dans un matériau de structure cristalline cubique.

un microscope électronique à balayage (MEB) [3, 4] est une technique qui permet la construction de cartographies d'orientations et le calcul de la FDOC localement. Elle permet donc de visualiser non seulement la taille et la forme des grains, mais aussi leur orientation cristallographique (schématisée dans la figure 1 par un cube dans le cas d'une structure cubique).

\section{Description et représentation des orientations cristallines}

Pour décrire une orientation cristalline, on définit une relation matricielle qui lie le repère du cristal à celui de l'échantillon par une matrice de rotation g [1]. Cette matrice peut être exprimée à partir de différents paramètres comme les indices de Miller $\{$ hkl $\}<$ uvw $>$. Le plan $\{h k l\}$ est 

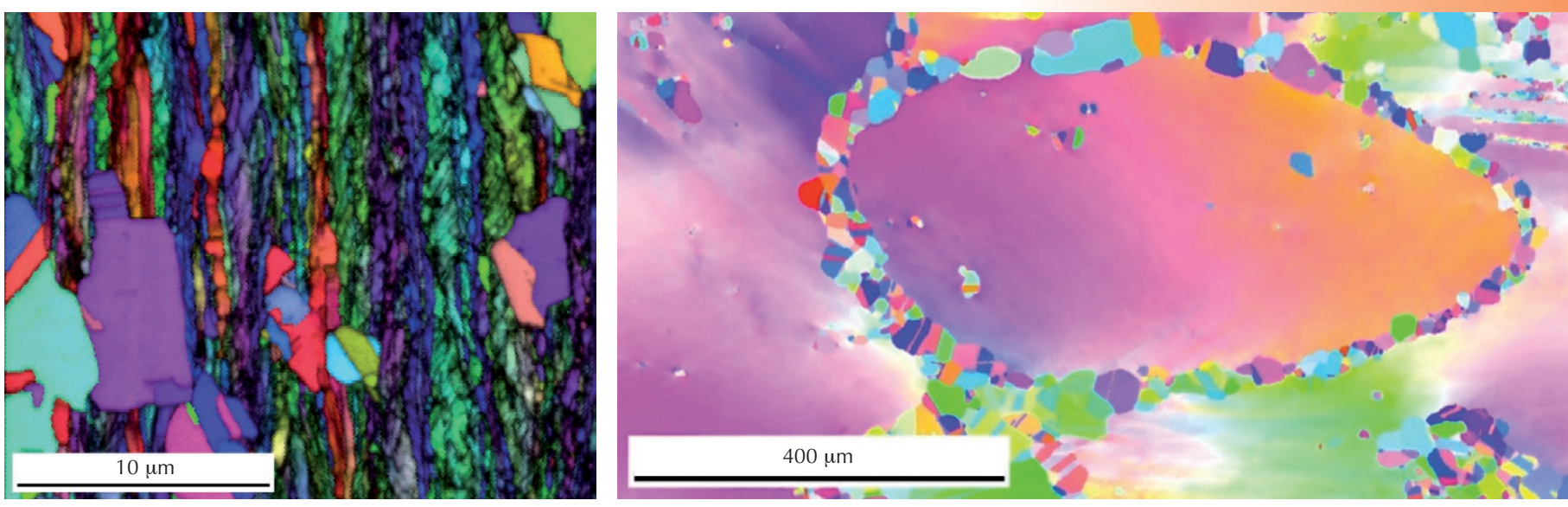

À gauche : cartographie EBSD d'orientations d’un alliage de fer-nickel partiellement recristallisé. Certains grains se sont développés aux dépens de la matrice déformée.

À droite : cartographie EBSD d'orientations après forgeage à chaud d'un alliage à base de nickel. 0n observe une recristallisation en collier : des grains ont recristallisé à l'aplomb de joints de grains déformés.

Dans ces deux cartographies, les différentes couleurs correspondent à différentes orientations cristallographiques.

parallèle au plan d'observation [par exemple le plan de laminage (DL : direction de laminage, DT : direction transverse) dans le cas d'une tôle], et la direction $<$ uvw $>$ est parallèle à une direction représentative de l'échantillon (par exemple, DL pour la tôle).

La figure de pôles directe est souvent utilisée pour décrire les orientations cristallines. Elle correspond à la projection stéréographique sur le plan de l'échantillon, de la distribution de la densité de pôles d'une famille de plans $\{\mathrm{hkl}\}$.

Considérons un cristal de structure cubique possédant une orientation donnée (fig. 2a). Les normales aux plans $\{100\}^{(1)}$ de ce cristal " percent » la sphère des pôles (sphère imaginaire centrée sur le grain considéré) en des points caractéristiques de l'hémisphère nord, dont la position est fonction de l'orientation du cristal dans le repère échantillon (DL, DT, DN). On peut alors utiliser le pôle sud comme pôle de projection de ces points sur le plan équatorial. On vient ainsi de réaliser une projection stéréographique pour obtenir la figure de pôles $\{100\}$ du cristal considéré (fig. 2b).

La figure $2 \mathrm{~b}$ montre un exemple de construction d'une figure de pôles $\{100\}$ correspondant à une orientation dite "Cube ", $\{001\}<100>$. Dans ce cas, le plan (001) de la famille de plans $\{100\}$ est parallèle au plan de la tôle (DL, DT) et, comme les indices du plan sont les
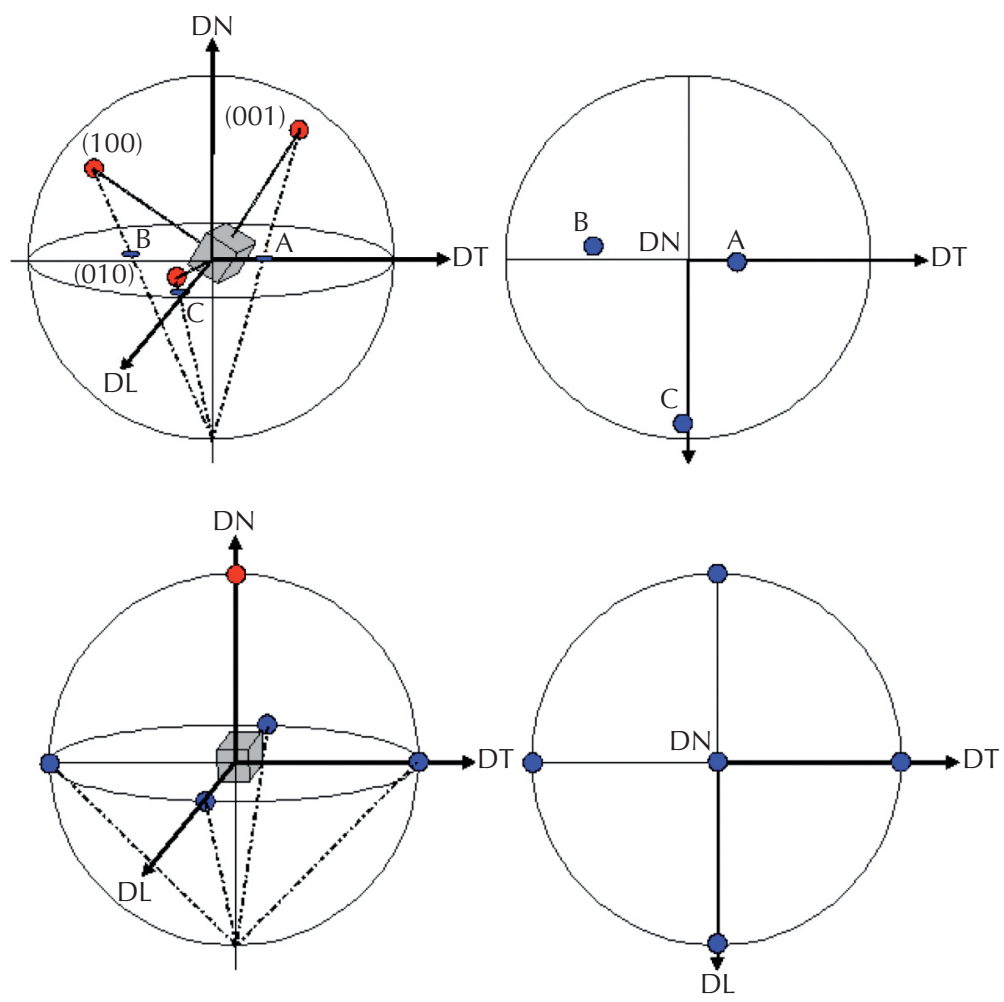

2. Construction d'une figure de pôles $\{100\}$, (a) pour une orientation quelconque et (b) pour une orientation Cube $\{001\}<100>$. À gauche : méthode de construction; à droite : figures de pôles obtenues.

mêmes que ceux de la normale au plan pour un cristal de structure cubique, la figure de pôles présente donc une direction $<001>$ parallèle à la direction normale au plan de la tôle, DN. Par ailleurs, il existe également une direction de la famille $<100>$ parallèle à DL et une autre parallèle à DT.

À chaque plan cristallin $\{$ hkl $\}$ d'un grain est associée une figure de pôles, et la figure de pôles $\{h \mathrm{hl}\}$ globale d'un échantillon polycristallin est la superposition des figures de pôles $\{\mathrm{hkl}\}$ des différents grains pondérées par leurs fractions volumiques. Pour caractériser la texture d'un échantillon, on est donc amené à mesurer plusieurs figures de pôles $\{h \mathrm{kl}\}$ par diffraction des rayons $\mathrm{X}$ ou des neutrons. Dans le cas de l'EBSD, on mesure l'orientation des grains et on trace des figures de pôles pour analyser la texture globale du polycristal. 


\section{Détermination de la texture}

\section{Approches globales}

La diffraction des rayons $\mathrm{X}$ et des neutrons permet de mesurer des figures de pôles directes $\{h k l\}$ [2]. Ces figures sont obtenues à partir de la mesure de l'intensité diffractée selon des cercles et des rayons caractéristiques. En effet, l'échantillon est soumis à deux rotations (rotation azimutale $\varphi$ et déclinaison $\chi$ ) (fig. 3) pour amener successivement tous les plans cristallins $\{h k l\}$ à diffracter.

Une analyse quantitative de la texture nécessite la mesure de plusieurs figures de pôles à des angles de Bragg $(\theta, 2 \theta)$ différents. D'une manière générale, dans le cas de la diffraction des rayons $\mathrm{X}$, les mesures sont réalisées en réflexion jusqu'à un angle de déclinaison $\chi$ de $70-80^{\circ}$. Au-delà, on se heurte à des problèmes de défocalisation, compte tenu du basculement de l'échantillon. Au contraire, en diffraction des neutrons où l'on travaille en transmission [1], ce phénomène est absent et les figures de pôles mesurées sont complètes.

Les volumes sondés sont généralement de quelques $10^{-3} \mathrm{~cm}^{3}$ par diffraction de rayons X d'énergie moyenne, et de l'ordre $\mathrm{du} \mathrm{cm}^{3}$ par diffraction des neutrons. Ces derniers permettent donc d'avoir une bonne statistique de comptage, de travailler sur des matériaux à gros grains et d'accéder à des composantes de la texture en faible fraction volumique. En revanche, les rayons $\mathrm{X}$ permettent de caractériser des gradients de texture.

\section{Approches locales}

Dans cette partie nous nous limiterons à la description de l'EBSD [3], qui est maintenant un outil couramment employé dans les laboratoires universitaires et industriels pour caractériser simultanément la microstructure et la texture en surface d'un échantillon polycristallin. Notons qu'il existe d'autres approches pour mesurer localement une orientation cristallographique, comme la microscopie en transmission ou la microdiffraction Laue qui utilise une source synchrotron de rayons $\mathrm{X}[5]$.

L'EBSD est installé dans un MEB possédant, le plus souvent, un canon à émission de champ (Field Emission Gun - FEG) qui permet d'atteindre une résolution de 20 à $50 \mathrm{~nm}$.

Sous l'effet des interactions élastiques et inélastiques avec les atomes de l'échantillon, les électrons incidents sont diffusés et ralentis dans celui-ci. Une fraction d'entre eux est rétrodiffusée hors de la cible, cette fraction étant d'autant plus importante que le nombre atomique de la cible est élevé et que l'angle d'inclinaison est grand. Avant de ressortir de l'échantillon, une proportion minoritaire de ces électrons rétrodiffusés peut avoir subi la diffraction de Bragg sur certaines familles de plans cristallins. Ces électrons, diffractés par une famille de plans donnée, forment deux cônes de diffraction très ouverts compte tenu de la faible valeur de l'angle de Bragg (de l'ordre du degré) due à la faible longueur d'onde (environ 0,087 $\AA$ pour une tension de $20 \mathrm{kV}$ ). L'intersection des deux cônes par un écran de phosphore, placé devant l'échantillon, donne lieu à une paire de "lignes de Kikuchi " qui délimitent une bande (fig. 4a). Cette bande correspond donc à la trace des plans diffractants (fig. 4c) sur l'écran. Pour une région du cristal, sur laquelle est focalisé le faisceau, la diffraction des électrons rétrodiffusés se produit sur plusieurs familles de plans cristallins. Il y a alors formation de plusieurs bandes, qui constituent le diagramme de Kikuchi (fig. 4b). Il est à noter que pour favoriser le rendement de rétrodiffusion et donc pour optimiser la qualité des diagrammes de Kikuchi, il a été montré que l'échantillon devait être incliné de $70^{\circ}$. La surface de l'échantillon doit être parfaitement plane, et comme la profondeur de pénétration est de l'ordre de quelques dizaines de nanomètres, une préparation soignée de cette surface est nécessaire. En particulier, tout écrouissage de surface dû au polissage mécanique doit être supprimé, par exemple à l'aide d'un polissage électrolytique.

C'est à partir de ce diagramme de Kikuchi que l'orientation cristallographique du volume analysé est déterminée, en se basant sur des considérations géométriques liées à la position des bandes. Cette indexation est entièrement automatisée. De plus, comme on peut piloter le faisceau et/ou la platine motorisée qui supporte l'échantillon, il est possible de mesurer un grand nombre de points et d'établir des cartographies d'orientations, qui permettent une analyse fine et simultanée de la microstructure (taille et forme des grains, phases...), de la microtexture ainsi que de la nature des joints de grains.
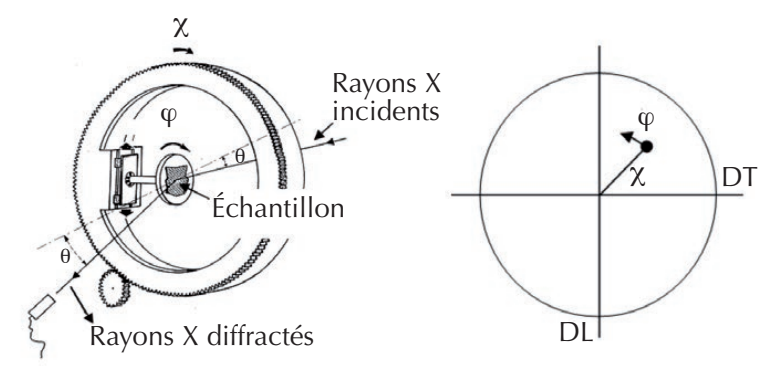

3. Principe de la mesure d'une figure de pôles $\{h k l\}$ d'un polycristal par diffraction des rayons $\mathrm{X}$. À gauche : dispositif expérimental. À droite : projection stéréographique.

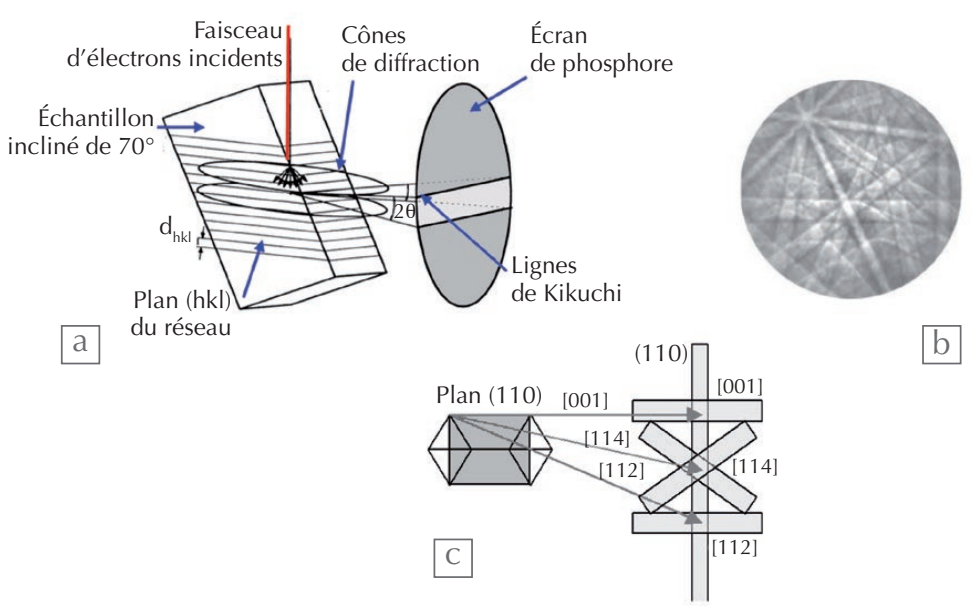

4. Lignes de Kikuchi. (a) Schéma montrant le dispositif pour une observation EBSD. (b) Exemple de diagramme de Kikuchi. (c) Correspondance entre un cristal (à gauche) et les bandes de Kikuchi (à droite). 


\section{Analyse quantitative de la texture}

\section{Approches globales}

Le principe de cette analyse consiste à résoudre l'équation fondamentale (équation 1) qui lie la FDOC aux figures de pôles expérimentales (densités de pôles) mesurées par diffraction des rayons $\mathrm{X}$ ou des neutrons. Pour une famille de plans $\{h k l\}$, la densité de pôles $\left(\mathrm{P}_{\mathrm{hkl}}\right)$ en un point $(\chi, \varphi)$, d'une figure de pôles s'exprime donc en fonction de la FDOC $\mathrm{F}(\mathrm{g})$ par la relation suivante

$$
P_{h k l}(\chi, \varphi)=\frac{1}{2 \pi} \int_{0}^{2 \pi} F(g) d \gamma
$$

Il existe plusieurs méthodes mathématiques (Harmonique, WIMV, ADC...) pour calculer $\mathrm{F}(\mathrm{g})$ [2, 6]. Toutefois, dans l'étude des textures cristallographiques à partir de la diffraction, il faut parfois prendre en compte les contraintes résiduelles élastiques qui subsistent dans de nombreux matériaux, comme ceux qui ont subi une mise en forme. De plus, le faisceau diffracté est également affecté par les réflectivités des diverses phases (échantillons polyphasés), la géométrie de l'échantillon dans le cas notamment de multicouches de différentes phases, ainsi que par la microstructure (taille et forme des cristallites, défauts). Depuis une décennie, une nouvelle approche globale associe toutes ces contributions multiples sous le nom d'analyse combinée. Elle a abouti au programme « Material Analysis Using Diffraction MAUD » [7-9], dont l'algorithme est donné dans la figure 5 .

\section{Approches locales}

Dans le cas de mesures d'orientations individuelles, on peut également calculer la FDOC (voir [2]) à l'aide de la méthode harmonique, chaque orientation étant modélisée par une gaussienne (ou une lorentzienne). Dans tous les cas, il convient de définir la largeur à mi-hauteur de la gaussienne ainsi que le nombre minimum d'orientations pour avoir une texture statistiquement admissible, c'est-à-dire comparable à celle mesurée par diffraction des rayons X. Cette largeur peut être reliée à un paramètre caractérisant l'acuité de la texture (voir [2]) et au nombre d'orientations individuelles mesurées qui lui-même est fonction de cette acuité. Pour une texture moyennement accusée, il a été montré qu'une centaine de grains permettait une description qualitative de la texture, mais qu'il en fallait au moins dix fois plus pour avoir une description quantitative avec une largeur de gaussienne de l'ordre de $5^{\circ}[1]$.

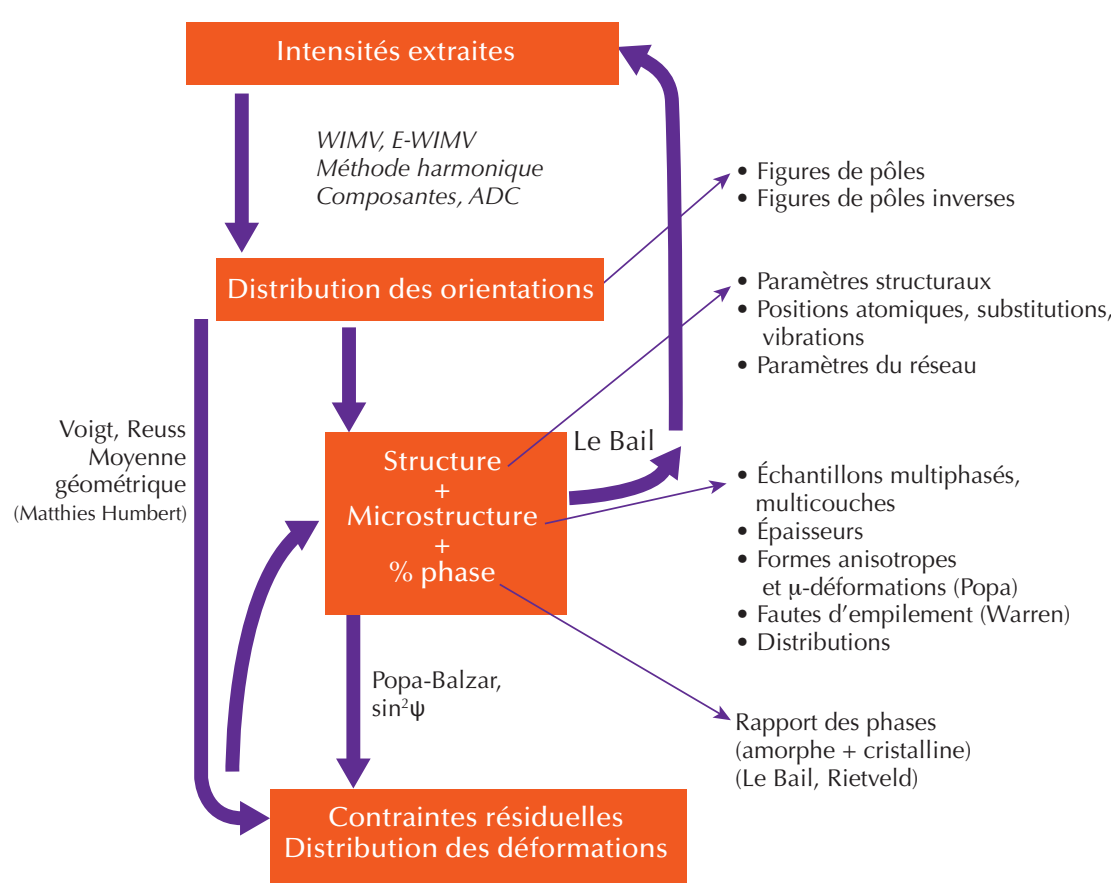

5. Programme MAUD. Algorithme des boucles imbriquées d'optimisation combinée sur l'ensemble des paramètres. La liste non limitative des paramètres pris en compte est donnée ci-dessus : analyse de texture, de structure, de tailles de cristallites, de cristallinité, de contraintes résiduelles... [7]

\section{Applications}

Augmentation de $1^{\prime}$ effet mémoire de forme d'un alliage Ni-Mn-Ga par texturation sous $\mathbf{l}^{\prime}$ 'effet d'un traitement thermomécanique Les alliages à mémoire de forme ferromagnétiques Ni-Mn-Ga sont des matériaux « intelligents », utilisés pour faire des capteurs et des actionneurs. À la température ambiante ils sont, pour une large gamme de composition, en phase martensitique ferromagnétique, de structure cristalline monoclinique, quasi orthorhombique. Sous l'action d'un champ magnétique, les " cristaux " de martensite se réorientent, ce qui entraîne des déformations géantes de plusieurs pourcents (jusqu'à 10\%).

La fabrication d'alliages polycristallins est beaucoup plus simple et moins coûteuse, mais dans ce cas l'orientation cristallographique quasi aléatoire des " cristaux " de martensite réduit fortement l'effet de mémoire de forme.

La texturation par traitement thermomécanique s'est révélée efficace pour restaurer l'effet mémoire de forme. Cette technique est illustrée par diffraction de neutrons in situ (diffractomètre STRESSSPEC, Centre de diffraction neutroniqueMLZ, Garching/Munich, Allemagne) pour la réorientation des " cristaux "

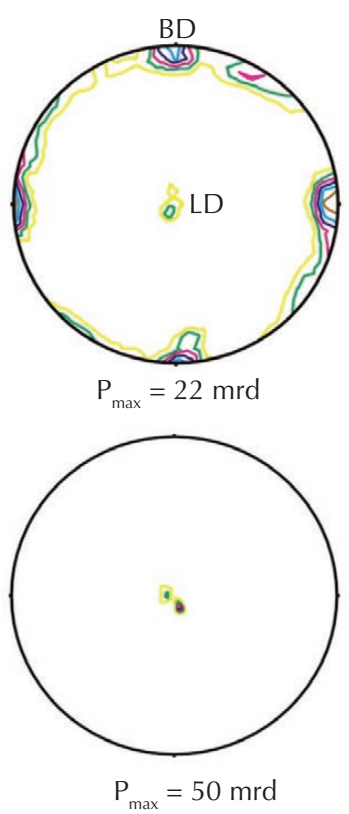

6. Figures de pôles (020) de l'échantillon de Ni-Mn-Ga. (a) À l'état brut de coulée.

(b) Après trois cycles successifs de traitement thermomécanique. Les lignes de niveau sont en multiples de densité aléatoire (mrd). $P_{\max }$ est l'intensité maximale du pic dans la figure de pôles, en unités mrd. 

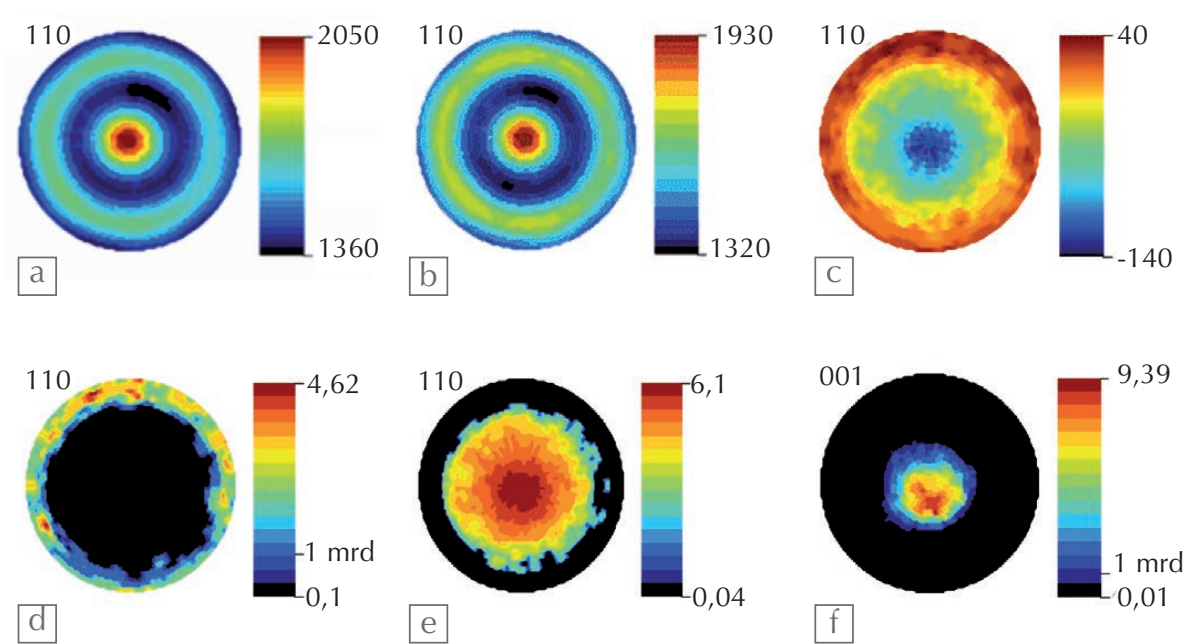

9,39 $\mathrm{mrd}$ f
7. Résultats de l'analyse quantitative de texture magnétique présentés sous la forme de figures de pôles directes mesurées sans et avec un champ magnétique appliqué, ainsi que sous la forme de figures de pôles issues de la diffraction magnétique [7]. a-c) Figures de pôles $\{110\}$ mesurées sans et avec un champ magnétique appliqué, et figure de pôles $\{110\}$ différence ou figure de pôles de polarisation.

d-e) Figures de pôles $\{110\}$ de diffraction magnétique pour les parties positive et négative de la polarisation. f) Figure de pôles $\{001\}$ recalculée à partir de la diffraction magnétique, qui illustre la réorientation des moments magnétiques dans la direction de l'axe de l'échantillon cylindrique sous l'effet du champ magnétique statique appliqué.

\〉

martensitiques - par traitement thermomécanique - dans un petit cylindre de $\mathrm{Ni-Mn-Ga} \mathrm{polycristallin} \mathrm{préparé} \mathrm{par}$ solidification directionnelle. Une compression de $10 \mathrm{MPa}$ (cycle 1), $25 \mathrm{MPa}$ (cycle 2) et $50 \mathrm{MPa}$ (cycle 3), respectivement, a été appliquée in situ parallèlement à la direction de solidification (LD, parallèle à l'axe du cylindre), au cours de la transformation martensitique à partir de la phase austénitique de structure cubique à faces centrées, lors du refroidissement dans le faisceau de neutrons dont l'incidence (BD) est perpendiculaire à l'axe du cylindre [10]. Les résultats montrent qu'avant traitement thermomécanique, les pics $<010>$ sur la figure de pôles (020) sont perpendiculaires ou parallèles à la direction de solidification LD (fig. 6a). Après trois cycles de traitement, il ne subsiste plus qu'un pic $<010>$ très intense au centre de la figure de pôles (fig. 6b). Ainsi, la mise en place d'une forte texturation dans laquelle les directions cristallines $<010>$ sont quasi parallèles à l'axe du cylindre (donc à la direction de compression LD), a pu être observée in situ au cours du traitement thermomécanique.

\section{Un exemple d'application}

de l'analyse combinée :

étude de textures magnétiques

L'analyse combinée est plus précise que les analyses de texture classiques. Cette méthode est donc bien adaptée à l'étude d'effets fins comme l'anisotropie de la polarisation magnétique. Les spectres de diffraction de neutrons présentent, en plus des raies de diffraction nucléaire (diffraction par les noyaux des atomes) des pics de diffraction magnétique, qui résultent de l'interaction entre les spins des neutrons et les moments magnétiques des matériaux. Le principe général de l'analyse quantitative de texture magnétique a été développé récemment [7]. Deux analyses successives sont effectuées sur un petit cylindre de fer, avec et sans application parallèlement à l'axe du cylindre d'un champ magnétique statique $\mathrm{B}$ de 0,3 T. Dans ce but, un porteéchantillon spécifique a été développé pour permettre de réaliser les deux mesures consécutives sans démonter l'échantillon cylindrique de son support. Dans le cas considéré d'un échantillon ferromagnétique solide, le seul effet du champ magnétique est d'orienter les moments magnétiques de l'échantillon, c'est-à-dire d'induire une polarisation magnétique représentée par un terme $\Delta \mathrm{P}^{\mathrm{m}}$. L'intensité diffractée des figures de pôles $\mathrm{P}_{\{h k 1\}}(\mathbf{y}, \mathrm{B})$ peut alors s'écrire sous la forme simplifiée :

$$
\begin{gathered}
\mathrm{P}_{\{\mathrm{hkl}\}}(\mathbf{y}, \mathrm{B})=\mathrm{P}_{\{\mathrm{hkl}\}}(\mathbf{y})+\mathrm{P}^{\mathrm{m}}{ }_{\{\mathrm{hkl}\}}(\mathbf{y}) \\
+\Delta \mathrm{P}^{\mathrm{m}}{ }_{\{\mathrm{hkl}\}}(\mathbf{y}, \mathrm{B}) \quad(2)
\end{gathered}
$$

où $\mathbf{y}=(\chi, \varphi)$ est la direction représentée en projection stéréographique dans la figure 3 et les indices supérieurs $n$ et $m$ signifient respectivement "nucléaire " et " magnétique ".

La contribution purement nucléaire $\mathrm{P}_{\{h k 1\}}^{\mathrm{n}}(\mathbf{y})$ provient de la diffraction des noyaux atomiques et correspond à la figure de pôles traditionnelle décrite sur la figure 2. Elle est liée à la FDOC F(g) par l'équation (1). Elle peut également être déterminée par diffraction des rayons $\mathrm{X}$ suivie d'un calcul de FDOC par l'une des méthodes d'inversion de figures de pôles.

La figure de pôles $\mathrm{P}_{\{\mathrm{mkl}\}}(\mathbf{y})$ représente l'intensité de diffraction magnétique. La figure de pôles de polarisation magnétique $\Delta \mathrm{P}_{\{\mathrm{hkl}\}}(\mathbf{y}, \mathrm{B})$ décrit la réorientation des moments magnétiques de l'échantillon ferromagnétique sous l'effet du champ magnétique appliqué (fig. 7c). Elle est la différence entre les figures de pôles mesurées sans et avec champ magnétique appliqué (figs. $7 \mathrm{a}$ et $7 \mathrm{~b}$ ). Alors que les figures de pôles cristallographiques sont toujours positives, les figures de pôles de polarisation magnétique peuvent être séparées en partie positive (fig. $7 \mathrm{~d}$ ) et partie négative de la polarisation (fig. 7e), comme illustré pour la figure de pôles de diffraction magnétique $\{110\}$. Il était intéressant de représenter aussi la figure de pôles - recalculée - de diffraction magnétique $\mathrm{P}_{\{001\}}^{\mathrm{m}}$. Cette figure, où l'on observe essentiellement un pic au centre (fig. 7f), illustre la réorientation des moments magnétiques parallèlement à l'axe de l'échantillon cylindrique, c'està-dire au champ magnétique appliqué.

\section{Hypertexturation Cube d'alliages} de Fe-Ni et de Ni-W

La fabrication à bas coût de cellules photovoltaïques pour obtenir une énergie respectueuse de l'environnement, nécessite d'élaborer des substrats métalliques hypertexturés Cube $\{100\}<001>$ afin de pouvoir y déposer par épitaxie des couches minces de silicium. La problématique est la même pour la production de câbles supraconducteurs, qui nécessite le dépôt en épitaxie de l'oxyde $\mathrm{YBaCuO}$ sur un substrat hypertexturé Cube (on vise 100\% de grains avec une orientation Cube $\{100\}<001>$ ). L'objectif de cette recherche consiste donc à développer un substrat d'alliage $\mathrm{Fe}-\mathrm{Ni}$ ou Ni-W avec des grains possédant tous l'orientation Cube, avec une très faible dispersion autour de 

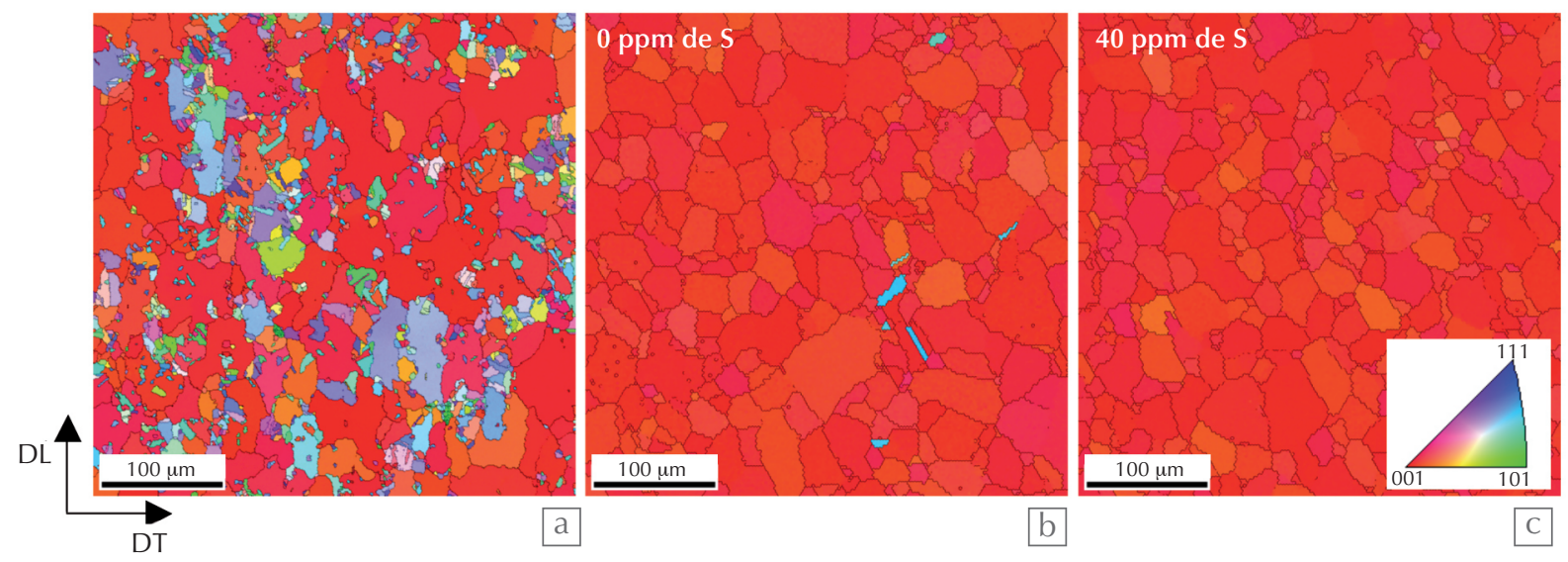

8. Augmentation de la texturation Cube d'un alliage de fer-nickel par ajout de soufre. Cartographies EBSD décrivant la distribution des plans \{hkl\} // (DL, DT) pour (a) un échantillon de Fe-48\%Ni laminé de $98 \%$ et recuit $1 \mathrm{~h}$ à $600^{\circ} \mathrm{C}$ et pour des coulées modèles contenant (b) 0 ppm et (c) 40 ppm de soufre, ayant subi un laminage de $99 \%$ et un traitement thermique optimisé [11]. Les joints de grains sont tracés en noir. Le code couleurs est donné sur la cartographie c.

l’orientation idéale. Pour accéder à cette texture accusée, il faut notamment fortement laminer la tôle (taux de réduction de 98-99\%). La figure 8a montre cependant que ce paramètre n'est pas suffisant, puisqu'après un laminage de $98 \%$ puis un recuit de recristallisation d'une heure à $600^{\circ} \mathrm{C}$, la fraction de grains Cube (rouges) avec une désorientation inférieure ou égale à $10^{\circ}$ n'est que de $74 \%$. Il est donc nécessaire d'optimiser le traitement thermique. En particulier, la température de recuit doit être suffisamment élevée (environ $1000^{\circ} \mathrm{C}$, selon les nuances chimiques) pour éliminer les macles $\{122\}<221>$ de l'orientation Cube, sans toutefois déclencher l'apparition de croissance anormale de grains. Il faut également ajuster au mieux la composition chimique du substrat. À titre d'exemple, dans le cadre d'une étude fondamentale, les figures $8 \mathrm{~b}-\mathrm{c}$ montrent les cartographies d'orientations obtenues sur des substrats de $\mathrm{Fe}-48 \% \mathrm{Ni}$ avec deux taux de soufre différents [11].

Ces résultats montrent clairement que la fraction de grains Cube avec une désorientation inférieure ou égale à $10^{\circ}$, augmente avec le taux de soufre $(98,4 \%$, $99,0 \%$ et $99,9 \%$, pour respectivement 0 , 20 et $40 \mathrm{ppm}$ de soufre), et atteint pratiquement $100 \%$ pour une teneur de $40 \mathrm{ppm}$.

D'après les auteurs [11], l'introduction de soufre induit la formation de précipités MnS (0,3\% en poids de $\mathrm{Mn})$ qui sont à l'origine de l'augmentation de l'énergie élastique stockée par les grains au cours de la déformation. Cette énergie, qui est motrice pour la recristallisation, a été mesurée par diffraction des neutrons (mesure de figures de pôles d'élargissement de pics de diffraction [1]) pour les composantes principales de la texture. Il apparaît effectivement que l'énergie stockée augmente avec le taux de soufre pour toutes les autres composantes de texture induites par la déformation $(\{110\}<112>,\{112\}<111>$ et $\{123\}<634>)$. En revanche, elle reste quasi constante dans les grains Cube. Autrement dit, les précipités de $\mathrm{MnS}$ ont un rôle minime sur l'écrouissage de ces grains, ce qui pourrait être dû au plus faible nombre de systèmes de glissement activés. Finalement, l'augmentation du taux de soufre accentue la différence d'énergie stockée entre la composante Cube et les autres composantes de déformation et facilite donc son développement. Notons que le taux de soufre doit cependant être limité pour d'autres raisons métallurgiques.

D'autres éléments chimiques ont également été étudiés comme l'aluminium, le bore, le titane et le zirconium [11]. Il n'apparaît pas d'effets significatifs sur le développement de la texture Cube. Au contraire, le niobium tend à inhiber son développement.

\section{Conclusion}

La texture cristallographique est un paramètre qui doit être maîtrisé, tout comme la microstructure, si l'on souhaite optimiser les propriétés d'usage des matériaux. Différentes techniques de diffraction, très complémentaires car ne travaillant pas à la même échelle, permettent de la déterminer. Les applications ne se limitent pas aux matériaux métalliques, puisque tous les matériaux cristallins peuvent être analysés à l'aide de ces techniques expérimentales.

\section{Références}

1- Rayonnement synchrotron, rayons $X$ et neutrons au service des matériaux - Analyse des contraintes et des textures, Eds. A. Lodini et T. Baudin, EDP Sciences (2012), pp. 278-302.

2- C. Esling et H.J. Bunge, Texture et anisotropie des matériaux polycristallins - Défnitions et techniques expérimentales, Techniques de l'Ingénieur, M3040 (2012).

3• T. Baudin, Analyse EBSD - Principe et cartographies d'orientations, Techniques de l'Ingénieur, M4138 (2010).

4• T. Baudin, Analyse EBSD - Déformation et recristallisation des matériaux métalliques, Techniques de l'Ingénieur, M4139 (2011).

\section{5• 0. Ulrich et al., Rev. Sci. Instrum. 82, (2011) 033908.}

6• H.-J. Bunge, Texture Analysis in Materials Science: Mathematical Methods, Butterworths (1982).

7• D. Chateigner, Combined analysis, Wiley-ISTE (2010).

8• L. Lutterotti et al., Thin Solid Films 450 (2004) $34-41$.

9• D. Chateigner et al., J. Appl. Cryst. 30 (1997) 43-48. 10• Z.B. Li et al., Appl. Phys. Lett. 105 (2014) 021907.

11• Y. Ateba Betanda et al., Adv. Eng. Mater. 16 (2014) 933-939.

Remerciements

CE remercie Y. Zhang, IR HDR à l'université de Lorraine, Z. Li, MC à NEU Shenyang, Chine et W. Gan, Ingénieur au GEMS, Allemagne. DC remercie le Conseil Régional de Basse-Normandie et le FEDER pour le financement de la Chaire d'Excellence de LL. Les cartographies d'orientations décrites dans le paragraphe sur l'hypertexturation Cube sont issues des travaux de thèse de Y. Ateba Betanda (Université Paris-Sud, Orsay), que TB remercie pour son aide. 